\title{
Robustness of a Class of Three-Dimensional Curve Tracking Control Laws under Time Delays and Polygonal State Constraints
}

\author{
Michael Malisoff
}

curve tracking dynamics. However, generalizing [9] to 3D tracking controllers, such as those in [4], [17], is challenging.

Using the penalty function approach to $3 \mathrm{D}$ curve tracking control laws in [4], [17] (which were derived from a nonstrict Lyapunov function), this paper presents a strict Lyapunov function construction. Our strict Lyapunov function allows us to prove ISS of the 3D curve tracking dynamics with respect to controller uncertainty under input delays, with predictable tolerance and safety bounds, and is based on the general approach from [8] for converting a nonstrict Lyapunov function into a strict one. Our work is motivated by our recent deployment of marine robots during our search for remaining pollution from the Deepwater Horizon oil spill disaster, where we used the 2D curve tracking controllers from [9]. By extending that work to the more difficult 3D curve case, here we obtain certified tracking, safety, and tolerance results that are more amenable to the $3 \mathrm{D}$ curves that naturally arise in real life marine robotics applications. This note is a summary of some results from [10]; see [10] for proofs of all results to follow.

\section{3D CURVE Tracking CONTROL}

Trajectories traced by a pair of particles moving in 3D space were studied in [4], [17]. One particle moves freely, hence called the free particle. The other is confined to a specified 3D curve. The second particle has locally the smallest distance to the free particle, so we refer to the second particle as the closest point. We next present our controls that drive the free particle and the closest particle to move at constant distance, to achieve curve tracking.

Let $\mathbf{r}_{1}$ denote the position of the second particle (at the closest point), $\mathbf{x}_{1}$ the unit tangent vector to the curve at $\mathbf{r}_{1}, \mathbf{y}_{1}$ a unit normal vector, and $\mathbf{z}_{1}$ a binormal vector. The velocity of the point is in the direction of $\mathbf{x}_{1}$, and the speed is defined by $\frac{d s}{d t}=\alpha$. Let $\mathbf{r}_{2}$ denote the position of the free particle moving at unit speed, $\mathbf{x}_{2}$ the unit tangent vector to the trajectory of its moving center, $\mathbf{y}_{2}$ a corresponding unit normal vector, and $\mathbf{z}_{2}=\mathbf{x}_{2} \times \mathbf{y}_{2}$. The dynamics of the point on the curve and the free moving particle are then

$$
\begin{array}{ll}
\dot{\mathbf{r}}_{1}=\alpha \mathbf{x}_{1} & \dot{\mathbf{r}}_{2}=\mathbf{x}_{2} \\
\dot{\mathbf{x}}_{1}=\alpha \kappa_{n} \mathbf{y}_{1}+\alpha \kappa_{g} \mathbf{z}_{1} & \dot{\mathbf{x}}_{2}=u \mathbf{y}_{2}+v \mathbf{z}_{2} \\
\dot{\mathbf{y}}_{1}=-\alpha \kappa_{n} \mathbf{x}_{1} & \dot{\mathbf{y}}_{2}=-u \mathbf{x}_{2} \\
\dot{\mathbf{z}}_{1}=-\alpha \kappa_{g} \mathbf{x}_{1}, & \dot{\mathbf{z}}_{2}=-v \mathbf{x}_{2},
\end{array}
$$

Malisoff is with Department of Mathematics, Louisiana State University, 303 Lockett Hall, Baton Rouge, LA 70803-4918, malisoff@1su.edu. Zhang is with the School of Electrical and Computer Engineering, Georgia Institute of Technology, 85 Fifth Street NW, Atlanta, GA 30332-0250, fumin@gatech.edu. Malisoff was supported by NSF Grant ECCS-1102348 and the Roy Paul Daniels Professorship \#3 in the Louisiana State University College of Science. Zhang was supported by ONR Grants N00014-081-1007 and N00014-09-1-1074, and NSF Grants ECCS-0841195, ECCS0845333 (CAREER), ECCS-1056253, and CNS-0931576.

where $\kappa_{n}$ and $\kappa_{g}$ are called the natural curvatures, and where $u$ and $v$ are steering controls that will be designed. We call $\kappa_{n}$ the normal curvature and $\kappa_{g}$ the geodesic curvature, and we assume that both curvatures are $C^{1}$, bounded and nonpositive valued (but see [10] for analogs that allow positive valued 
curvatures). Later we put more specific conditions on the curvature functions. The control goal is for the distance $\mid \mathbf{r}_{2}-$ $\mathbf{r}_{1} \mid$ between the particles to converge to a desired positive constant distance, and to control $\mathbf{x}_{2}$ to be aligned with $\mathbf{x}_{1}$. We assume that $\alpha$ is nowhere zero. Later, we restrict the state space to be a forward invariant set where $\alpha$ is guaranteed to stay positive.

To design controls $u$ and $v$ for (1), we first perform a coordinate change to shape variables. A necessary condition for minimizing the distance between the free particle and the closest point is the orthogonality condition $\left(\mathbf{r}_{2}-\mathbf{r}_{1}\right) \cdot \mathbf{x}_{1}=$ 0 . This follows because for each $t$, the function $\mathscr{M}_{t}(h)=$ $\left|\mathbf{r}_{1}(t+h)-\mathbf{r}_{2}(t)\right|^{2}$ has a local minimum at $h=0$, so $\mathscr{M}_{t}^{\prime}(0)=$ $2 \alpha\left(\mathbf{r}_{2}(t)-\mathbf{r}_{1}(t)\right) \cdot \mathbf{x}_{1}(t)=0$ and $\alpha$ is nowhere zero. Hence, since $\left\{\mathbf{x}_{1}, \mathbf{y}_{1}, \mathbf{z}_{1}\right\}$ is an orthonormal set, we have $\mathbf{r}_{2}-\mathbf{r}_{1}=$ $\rho_{1} \mathbf{y}_{1}+\rho_{2} \mathbf{z}_{1}$ where $\rho_{1}=\left(\mathbf{r}_{2}-\mathbf{r}_{1}\right) \cdot \mathbf{y}_{1}$ and $\rho_{2}=\left(\mathbf{r}_{2}-\mathbf{r}_{1}\right)$. $\mathbf{z}_{1}$. When the free particle moves, we can use the necessary condition, the orthonormality of the frame $\left(\mathbf{x}_{1}, \mathbf{y}_{1}, \mathbf{z}_{1}\right)$, and the product rule to show that $\dot{\rho}_{1}=\left(\mathbf{x}_{2}-\alpha \mathbf{x}_{1}\right) \cdot \mathbf{y}_{1}-\alpha \kappa_{n}\left(\mathbf{r}_{2}-\right.$ $\left.\mathbf{r}_{1}\right) \cdot \mathbf{x}_{1}=\mathbf{x}_{2} \cdot \mathbf{y}_{1}$ and $\dot{\rho}_{2}=\left(\mathbf{x}_{2}-\alpha \mathbf{x}_{1}\right) \cdot \mathbf{z}_{1}-\alpha \kappa_{g}\left(\mathbf{r}_{2}-\mathbf{r}_{1}\right) \cdot \mathbf{x}_{1}=$ $\mathbf{x}_{2} \cdot \mathbf{z}_{1}$. We express the relative distance between the frames $\left(\mathbf{x}_{1}, \mathbf{y}_{1}, \mathbf{z}_{1}\right)$ and $\left(\mathbf{x}_{2}, \mathbf{y}_{2}, \mathbf{z}_{2}\right)$ using the shape variables $\varphi=\mathbf{x}_{1}$. $\mathbf{x}_{2}, \beta=\mathbf{y}_{1} \cdot \mathbf{x}_{2}$, and $\gamma=\mathbf{z}_{1} \cdot \mathbf{x}_{2}$. Then $\varphi^{2}+\beta^{2}+\gamma^{2}=1$. From the equation $\dot{\mathbf{x}}_{2}=u \mathbf{y}_{2}+v \mathbf{z}_{2}$, we get $u=\dot{\mathbf{x}}_{2} \cdot \mathbf{y}_{2}$ and $v=\dot{\mathbf{x}}_{2} \cdot \mathbf{z}_{2}$. Since $\mathbf{x}_{1}, \mathbf{y}_{1}$ and $\mathbf{z}_{1}$ form a basis of $\mathbb{R}^{3}, \dot{\mathbf{x}}_{2}$ can be expressed as a linear combination of $\mathbf{x}_{1}, \mathbf{y}_{1}$ and $\mathbf{z}_{1}$ as $\dot{\mathbf{x}}_{2}=a_{1} \mathbf{x}_{1}+a_{2} \mathbf{y}_{1}+$ $a_{3} \mathbf{z}_{1}$, where the scalars $a_{1}, a_{2}$ and $a_{3}$ depend on the dynamics of the particles. Hence,

$$
\begin{aligned}
& u=a_{1}\left(\mathbf{x}_{1} \cdot \mathbf{y}_{2}\right)+a_{2}\left(\mathbf{y}_{1} \cdot \mathbf{y}_{2}\right)+a_{3}\left(\mathbf{z}_{1} \cdot \mathbf{y}_{2}\right) \text { and } \\
& v=a_{1}\left(\mathbf{x}_{1} \cdot \mathbf{z}_{2}\right)+a_{2}\left(\mathbf{y}_{1} \cdot \mathbf{z}_{2}\right)+a_{3}\left(\mathbf{z}_{1} \cdot \mathbf{z}_{2}\right) .
\end{aligned}
$$

The design of $u$ and $v$ becomes finding the $a_{i}$ 's. Simple calculations [10] then give the open loop system dynamics

$$
\begin{aligned}
\dot{\rho}_{1} & =\beta \\
\dot{\rho}_{2} & =\gamma \\
\dot{\varphi} & =\alpha \kappa_{n} \beta+\alpha \kappa_{g} \gamma+a_{1}\left(1-\varphi^{2}\right)-a_{2} \varphi \beta-a_{3} \varphi \gamma \\
\dot{\beta} & =-\alpha \kappa_{n} \varphi-a_{1} \varphi \beta+a_{2}\left(1-\beta^{2}\right)-a_{3} \beta \gamma \\
\dot{\gamma} & =-\alpha \kappa_{g} \varphi-a_{1} \varphi \gamma-a_{2} \beta \gamma+a_{3}\left(1-\gamma^{2}\right)
\end{aligned}
$$

on $(0,+\infty)^{2} \times S^{2}$, where $S^{2}=\left\{p \in \mathbb{R}^{3}:|p|=1\right\}$ is the unit sphere centered at the origin in $\mathbb{R}^{3}$ and

$$
\alpha=\frac{\mathbf{x}_{2} \cdot \mathbf{x}_{1}}{1-\left(\mathbf{r}_{2}-\mathbf{r}_{1}\right) \cdot\left(\kappa_{n} \mathbf{y}_{1}+\kappa_{g} \mathbf{z}_{1}\right)}=\frac{\mathbf{x}_{2} \cdot \mathbf{x}_{1}}{1-\kappa_{n} \rho_{1}-\kappa_{g} \rho_{2}}
$$

when the $\rho_{i}$ 's are nonnegative. The speed formula (4) follows by differentiating the orthogonality condition $\left(\mathbf{r}_{2}-\mathbf{r}_{1}\right) \cdot \mathbf{x}_{1}=$ 0 with respect to $t$. The denominator in (4) is nowhere zero because we assumed that the curvatures are nonpositive valued. Later we restrict the dynamics to a forward invariant set where the $\rho_{i}$ 's stay positive. Given any desired positive values $\rho_{c i}$ for $\rho_{i}$ for $i=1,2$, our first control objective is to design $a_{1}, a_{2}$, and $a_{3}$ in (2) such that $\left(\rho_{c 1}, \rho_{c 2}, 1,0,0\right)$ is a globally asymptotically stable equilibrium for (3) on the state space $\mathscr{Z}=\left\{\left(\rho_{1}, \rho_{2}, \varphi, \beta, \gamma\right) \in(0,+\infty)^{2} \times S^{2}: \varphi>0\right\}$ (on which $\alpha$ is positive valued). To this end, take

$$
a_{1}=\mu, a_{2}=-h_{1}^{\prime}\left(\rho_{1}\right)+\frac{\alpha \kappa_{n}}{\varphi}, a_{3}=-h_{2}^{\prime}\left(\rho_{2}\right)+\frac{\alpha \kappa_{g}}{\varphi},
$$

where $\mu>0$ is any constant and the functions $h_{i}$ satisfy:

Assumption 1: For $i=1,2$, the function $h_{i}:(0,+\infty) \rightarrow$ $[0,+\infty)$ is $C^{1}$ and satisfies the following: (a) $h_{i}^{\prime}(\rho)(\rho-$ $\left.\rho_{c i}\right)>0$ for all $\rho>0$ except $\rho=\rho_{c i}$, (b) $\lim _{\rho \rightarrow 0+} h_{i}(\rho)=$ $\lim _{\rho \rightarrow+\infty} h_{i}(\rho)=+\infty$, and (c) $h_{i}\left(\rho_{c i}\right)=0$.

We next use the spherical coordinate transformation

$$
(\varphi, \beta, \gamma)=(\cos (\zeta) \cos (\theta),-\sin (\zeta) \cos (\theta), \sin (\theta))
$$

where $\zeta$ and $\theta$ are both valued in $(-\pi / 2, \pi / 2)$. Then $\beta / \varphi=$ $-\tan (\zeta)$. It follows from the relations $\dot{\gamma}=\cos (\theta) \dot{\theta}$ and $\dot{\zeta}=$ $-\cos ^{2}(\zeta)(\dot{\beta} \varphi-\beta \dot{\varphi}) / \varphi^{2}$ that (3) in the new state vector $Y=$ $\left(\rho_{1}, \zeta, \rho_{2}, \theta\right)$ with the choices $(5)$ is

$$
\begin{aligned}
\dot{\rho}_{1}= & -\sin (\zeta) \cos (\theta) \\
\dot{\zeta}= & -\frac{1}{\cos ^{2}(\theta)}\left[\alpha \kappa_{n} \sin ^{2}(\theta)-h_{1}^{\prime}\left(\rho_{1}\right) \cos (\zeta) \cos (\theta)\right. \\
& \left.+\alpha \kappa_{g} \sin (\theta) \sin (\zeta) \cos (\theta)+\mu \sin (\zeta) \cos (\theta)\right] \\
\dot{\rho}_{2}= & \sin (\theta) \\
\dot{\theta}= & \alpha \kappa_{g} \frac{\sin ^{2}(\zeta)}{\cos (\zeta)}-h_{2}^{\prime}\left(\rho_{2}\right) \cos (\theta)-\mu \cos (\zeta) \sin (\theta) \\
& +\left(-h_{1}^{\prime}\left(\rho_{1}\right)+\frac{\alpha \kappa_{n}}{\cos (\theta) \cos (\zeta)}\right) \sin (\zeta) \sin (\theta)
\end{aligned}
$$

on the state space

$$
\mathscr{X}=(0,+\infty) \times(-\pi / 2, \pi / 2) \times(0,+\infty) \times(-\pi / 2, \pi / 2),
$$

and our global asymptotic stabilization objective will be realized if we prove that (7) is globally asymptotically stable to its equilibrium $\mathscr{E}=\left(\rho_{c 1}, 0, \rho_{c 2}, 0\right)$ on $\mathscr{X}$. To this end, we choose the Lyapunov function candidate

$$
V(Y)=-\ln (\cos (\theta) \cos (\zeta))+h_{1}\left(\rho_{1}\right)+h_{2}\left(\rho_{2}\right) .
$$

The function (9) is analogous to the nonstrict Lyapunov function used in 2D tracking in [9]. Its term $\ln (\varphi)=$ $\ln (\cos (\theta) \cos (\zeta))$ helps align the moving direction of the free particle with the tangent vector at the closest point. Moreover, Assumption 1 ensures that $V(Y) \rightarrow+\infty$ as $\zeta$ or $\theta$ approach $\pm \pi / 2$, or as $\rho_{1}$ or $\rho_{2}$ approach 0 or $+\infty$. Also,

$$
\dot{V} \leq-\frac{\mu}{\cos (\theta) \cos (\zeta)}\left(1-\cos ^{2}(\theta) \cos ^{2}(\zeta)\right) .
$$

holds along all trajectories of (7) in $\mathscr{X}$. Therefore, $\mathscr{X}$ is forward invariant for (7) and we have the following, which follows from the LaSalle Invariance Principle: If $\kappa_{n}$ and $\kappa_{g}$ are $C^{1}$ nonpositive valued functions of $Y$, then (7) is globally asymptotically stable to $\mathscr{E}$ on $\mathscr{X}$.

\section{Constructing a Strict Lyapunov Function}

The inequality (10) implies that $V$ is a nonstrict Lyapunov function for (7). By nonstrictness (resp., strictness) of a Lyapunov function $V$, we mean that $\dot{V}$ is nonpositive (resp., negative definite) along all trajectories of (7). It follows from converse Lyapunov function theory that a strict Lyapunov function exists for the asymptotically stable closed loop dynamics (7), but it is still useful to construct a closed form strict Lyapunov function, since this makes it possible to prove ISS under controller uncertainty, input delays, and state constraints, and obtain closed form formulas for the comparison functions in the ISS estimate and adaptive tracking and parameter identification. See [1], [3] for the motivation for adaptiveness of controllers. We build a strict Lyapunov function for (7), which has an added advantage that it allows time varying curvatures that need not be periodic in time (and therefore covers cases that cannot be covered by the LaSalle argument). 
The work [8] gives a general Matrosov approach for transforming nonstrict Lyapunov functions into strict ones, using auxiliary functions. However, it may be difficult to find the required auxiliary functions. Here we find the required auxiliary functions that allow us to convert (9) into a strict Lyapunov function for (7) on our state space $\mathscr{X}$ from (8). To this end, we add the following assumption (but see [10] for analogous results with curvatures that can also take positive values):

Assumption 2: The $h_{i}$ 's are $C^{2}$ and satisfy Assumption 1 . Also: (A) There is an increasing $C^{1}$ function $\lambda:[0,+\infty) \rightarrow$ $[\mu,+\infty)$ such that $\lambda\left(h_{i}(\rho)\right) \geq 1+0.5 \mu^{2}+h_{i}^{\prime \prime}(\rho)$ for all $\rho>$ 0 and $i=1,2$, (B) there is a function $\Gamma \in \mathscr{K}_{\infty} \cap C^{1}$ such that $\Gamma\left(h_{i}(\rho)\right) \geq\left[h_{i}^{\prime}(\rho)\right]^{2}$ for all $\rho>0$ and $i=1,2$, and (C) $h_{i}^{\prime \prime}(\rho) \geq 0$ for all $\rho>0$ and $i=1,2$. The curvatures $\kappa_{n}$ and $\kappa_{g}$ are bounded nonpositive valued $C^{1}$ functions of $(t, Y)$.

The preceding assumptions are satisfied by the functions

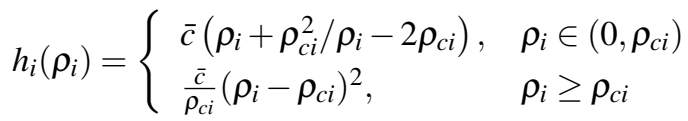

for any constant $\bar{c}>0$. To see why, recall from [9] that

$$
\begin{aligned}
& \lambda_{0}\left(q, \rho_{c i}\right)=\frac{2}{\bar{c}^{2} \rho_{c i}^{4}}\left(q+2 \bar{c} \rho_{c i}\right)^{3}+1+0.5 \mu^{2}+\mu \text { and } \\
& \Gamma_{0}\left(q, \rho_{c i}\right)=\frac{18 \bar{c}}{\rho_{c i}} q+\left(\frac{2}{\rho_{c i}}\right)^{4}\left(\frac{9}{\bar{c}^{2}}\right) q^{4}
\end{aligned}
$$

are such that $\lambda_{0}\left(h_{i}\left(\rho_{i}\right), \rho_{c i}\right) \geq 1+0.5 \mu^{2}+h_{i}^{\prime \prime}\left(\rho_{i}\right)$ and $\Gamma_{0}\left(h_{i}\left(\rho_{i}\right), \rho_{c i}\right) \geq\left[h_{i}^{\prime}\left(\rho_{i}\right)\right]^{2}$ for all $\rho_{i} \in\left(0, \rho_{c i}\right)$ for $i=1$ and $i=2$. Therefore, (11) satisfies Assumption 2 with the choices $\lambda(q)=\lambda_{0}\left(q, \rho_{c 1}\right)+\lambda_{0}\left(q, \rho_{c 2}\right)+2 \bar{c} / \min \left\{\rho_{c 1}, \rho_{c 2}\right\}$ and $\Gamma(q)=\Gamma_{0}\left(q, \rho_{c 1}\right)+\Gamma_{0}\left(q, \rho_{c 2}\right)+4 \bar{c} q / \min \left\{\rho_{c 1}, \rho_{c 2}\right\}$. Set

$$
\begin{aligned}
\mathscr{L}(q)= & \frac{3}{\mu}[\Gamma(q)+1]\left(1+\left\|\kappa_{n}\right\|_{\infty}+\left\|\kappa_{g}\right\|_{\infty}\right) \\
& +2\left[\frac{1}{\mu} \lambda(q)+\Gamma^{\prime}(q)+1\right],
\end{aligned}
$$

where $\mu$ is the steering constant from (5), and $\lambda$ and $\Gamma$ satisfy Assumption 2. In [10], we prove the following 3D analog of the key Lyapunov function construction from [9]:

Theorem 1: Let Assumption 2 hold and choose the nonstrict Lyapunov function $V$ from (9). Then

$$
\begin{aligned}
U(Y)= & -h_{1}^{\prime}\left(\rho_{1}\right) \sin (\zeta) \cos (\theta)+h_{2}^{\prime}\left(\rho_{2}\right) \sin (\theta) \\
& +\int_{0}^{V(Y)} \mathscr{L}(q) \mathrm{d} q
\end{aligned}
$$

is a strict Lyapunov function for the closed loop 3D curve tracking dynamics (7) on $\mathscr{X}=(0,+\infty) \times(-\pi / 2, \pi / 2) \times$ $(0,+\infty) \times(-\pi / 2, \pi / 2)$. Therefore, (7) is uniformly globally asymptotically stable to $\mathscr{E}=\left(\rho_{c 1}, 0, \rho_{c 2}, 0\right)$.

Sketch of Proof: Throughout the proof, all equalities and inequalities are over all trajectories of (7) in $\mathscr{X}$, so $\cos (\theta)$ and $\cos (\zeta)$ stay positive. By (10) and Assumption 2(C),

$$
\begin{aligned}
\dot{U} \leq & h_{1}^{\prime \prime}\left(\rho_{1}\right) \sin ^{2}(\zeta)-h_{1}^{\prime}\left(\rho_{1}\right) \cos (\zeta) \cos (\theta) \dot{\zeta} \\
& +h_{1}^{\prime}\left(\rho_{1}\right) \sin (\zeta) \sin (\theta) \dot{\theta} \\
& +h_{2}^{\prime \prime}\left(\rho_{2}\right) \sin ^{2}(\theta)+h_{2}^{\prime}\left(\rho_{2}\right) \cos (\theta) \dot{\theta} \\
& -\mathscr{L}(V(Y)) \frac{\mu\left(1-\cos ^{2}(\theta) \cos ^{2}(\zeta)\right)}{\cos (\theta) \cos (\zeta)}
\end{aligned}
$$

Setting $\quad J(Y)=-\left[h_{1}^{\prime}\left(\rho_{1}\right) \cos (\zeta)\right]^{2}-\left[h_{2}^{\prime}\left(\rho_{2}\right) \cos (\theta)\right]^{2}+$ $h_{1}^{\prime \prime}\left(\rho_{1}\right) \sin ^{2}(\zeta)+h_{2}^{\prime \prime}\left(\rho_{2}\right) \sin ^{2}(\theta)$, substituting the formulas for $\dot{\zeta}$ and $\dot{\theta}$ from (7) into (15), rearranging terms, and then dropping the nonpositive term $-\left[h_{1}^{\prime}\left(\rho_{1}\right) \sin (\zeta) \sin (\theta)\right]^{2}$ gives

$$
\begin{aligned}
\dot{U} \leq & J(Y)+h_{1}^{\prime}\left(\rho_{1}\right) \mu \cos (\zeta) \sin (\zeta) \cos ^{2}(\theta) \\
- & h_{2}^{\prime}\left(\rho_{2}\right) \mu \cos (\theta) \cos (\zeta) \sin (\theta) \\
+ & \left\{\frac { h _ { 1 } ^ { \prime } ( \rho _ { 1 } ) \operatorname { c o s } ^ { 2 } ( \zeta ) } { \operatorname { c o s } ( \theta ) \operatorname { c o s } ( \zeta ) } \left[\alpha \kappa_{n}\left(1-\cos ^{2}(\theta)\right)\right.\right. \\
& \left.+\alpha \kappa_{g} \sin (\theta) \sin (\zeta) \cos (\theta)\right] \\
& +\frac{h_{1}^{\prime}\left(\rho_{1}\right) \sin (\zeta) \sin (\theta)}{\cos (\theta)}\left[\frac{\alpha \kappa_{n}}{\cos (\zeta)} \sin (\zeta) \sin (\theta)\right. \\
& \left.+\frac{\alpha \kappa_{g}\left(1-\cos ^{2}(\zeta)\right)}{\cos (\zeta)} \cos (\theta)\right] \\
& -h_{1}^{\prime}\left(\rho_{1}\right) \sin (\zeta) \sin (\theta) \cos (\theta) h_{2}^{\prime}\left(\rho_{2}\right) \\
& +h_{2}^{\prime}\left(\rho_{2}\right)\left[\alpha \kappa_{g} \frac{\cos ^{2}(\theta)\left(1-\cos ^{2}(\zeta)\right)}{\cos (\theta) \cos (\zeta)}+\left(-h_{1}^{\prime}\left(\rho_{1}\right)\right.\right. \\
& \left.\left.\left.+\frac{\alpha \kappa_{n}}{\cos (\theta) \cos (\zeta)}\right) \sin (\theta) \cos (\theta) \sin (\zeta)\right]\right\} \\
& -\mathscr{L}(V(Y)) \frac{\mu\left(1-\cos ^{2}(\theta) \cos ^{2}(\zeta)\right)}{\cos (\theta) \cos (\zeta)} .
\end{aligned}
$$

The quantity in braces in (16) is bounded above by [10]

$$
3[\Gamma(V(Y))+1]\left(1+\left\|\kappa_{n}\right\|_{\infty}+\left\|\kappa_{g}\right\|_{\infty}\right) \frac{1-\cos ^{2}(\theta) \cos ^{2}(\zeta)}{\cos (\theta) \cos (\zeta)}
$$

on $\mathscr{X}$ (where $\|\cdot\|_{\infty}$ is the supremum), so (13) gives

$$
\begin{aligned}
\dot{U} \leq & -\left[h_{1}^{\prime}\left(\rho_{1}\right) \cos (\zeta)\right]^{2}+h_{1}^{\prime \prime}\left(\rho_{1}\right) \sin ^{2}(\zeta) \\
& +\left|h_{1}^{\prime}\left(\rho_{1}\right) \sin (\zeta)\right| \mu \cos (\zeta) \\
& -\left[\frac{1}{\mu} \lambda(V)+\Gamma^{\prime}(V)+1\right] \frac{\mu \sin ^{2}(\zeta)}{\cos (\zeta)} \\
& -\left[h_{2}^{\prime}\left(\rho_{2}\right) \cos (\theta)\right]^{2}+h_{2}^{\prime \prime}\left(\rho_{2}\right) \sin ^{2}(\theta) \\
& +\left|h_{2}^{\prime}\left(\rho_{2}\right) \sin (\theta)\right| \mu \cos (\theta) \\
& -\left[\frac{1}{\mu} \lambda(V)+\Gamma^{\prime}(V)+1\right] \frac{\mu \sin ^{2}(\theta)}{\cos (\theta)} .
\end{aligned}
$$

Also, the triangle inequality gives the following on $\mathscr{X}$ :

$\left|h_{1}^{\prime}\left(\rho_{1}\right) \sin (\zeta)\right| \cos (\zeta) \mu \leq \frac{1}{2}\left[h_{1}^{\prime}\left(\rho_{1}\right) \cos (\zeta)\right]^{2}+\frac{1}{2} \mu^{2} \sin ^{2}(\zeta)$

$\left|h_{2}^{\prime}\left(\rho_{2}\right) \sin (\theta)\right| \cos (\theta) \mu \leq \frac{1}{2}\left[h_{2}^{\prime}\left(\rho_{2}\right) \cos (\theta)\right]^{2}+\frac{1}{2} \mu^{2} \sin ^{2}(\theta)$

Combining this with (18) and recalling our condition $\lambda(V(Y)) \geq \lambda\left(h_{i}\left(\rho_{i}\right)\right) \geq 1+0.5 \mu^{2}+h_{i}^{\prime \prime}\left(\rho_{i}\right)$ from Assumption 2 and the facts that $\cos (\zeta) \in(0,1]$ and $\cos (\theta) \in(0,1]$ on our state space $\mathscr{X}$, we get the strict Lyapunov decay condition

$$
\begin{aligned}
\dot{U} \leq & -0.5\left[h_{1}^{\prime}\left(\rho_{1}\right) \cos (\zeta)\right]^{2}-\sin ^{2}(\zeta) \\
& -0.5\left[h_{2}^{\prime}\left(\rho_{2}\right) \cos (\theta)\right]^{2}-\sin ^{2}(\theta) .
\end{aligned}
$$

Then one can show that $U$ is proper and positive definite [10], meaning there are $\mathscr{K}_{\infty}$ functions $\underline{\alpha}$ and $\bar{\alpha}$ such that $\underline{\alpha}(|Y-\mathscr{E}|) \leq U(Y) \leq \bar{\alpha}(|Y-\mathscr{E}|)$ for all $Y \in \mathscr{X}$. Hence, $U$ is a strict Lyapunov function for (7) on $\mathscr{X}$.

\section{ISS AND ROBUST FORWARD INVARIANCE}

Using our strict Lyapunov function (14), it is natural to try to prove ISS of the perturbed version

$$
\begin{aligned}
\dot{\rho}_{1}= & -\sin (\zeta) \cos (\theta) \\
\dot{\zeta}= & -\frac{1}{\cos ^{2}(\theta)}\left[\alpha \kappa_{n} \sin ^{2}(\theta)-h_{1}^{\prime}\left(\rho_{1}\right) \cos (\zeta) \cos (\theta)\right. \\
& \left.+\sin (\zeta) \cos (\theta)\left(\alpha \kappa_{g} \sin (\theta)+\mu\right)\right]+\delta_{1}(t) \\
\dot{\rho}_{2}= & \sin (\theta) \\
\dot{\theta}= & \alpha \kappa_{g} \frac{\sin ^{2}(\zeta)}{\cos (\zeta)}-h_{2}^{\prime}\left(\rho_{2}\right) \cos (\theta)-\mu \cos (\zeta) \sin (\theta) \\
& +\left(-h_{1}^{\prime}\left(\rho_{1}\right)+\frac{\alpha \kappa_{n}}{\cos (\theta) \cos (\zeta)}\right) \sin (\zeta) \sin (\theta)+\delta_{2}(t)
\end{aligned}
$$


of (7) when there are perturbations $\delta=\left(\delta_{1}, \delta_{2}\right)$, representing uncertainty. See [9] where we used a strict Lyapunov function to prove ISS for the simpler 2D tracking case. The relevant definitions are as follows, where $\|\cdot\|_{S}$ is the (essential) supremum over any set $S,\|f\|_{\infty}$ is the supremum over the full domain of any function $f$, and $\mathscr{M} \mathscr{E} \mathscr{B}(\mathscr{D})$ is the set of all measurable essentially bounded functions $\delta:[0,+\infty) \rightarrow \mathscr{D}$ for each closed subset $\mathscr{D} \subseteq \mathbb{R}^{2}$.

By ISS of a system $\dot{z}=\mathscr{G}(t, z, \delta)$ on a subset $\mathscr{S}$ of its state space for a disturbance set $\mathscr{D}$ and an equilibrium $\mathscr{E}_{0} \in \mathscr{S}$, we mean that there are functions $\beta \in \mathscr{K} \mathscr{L}$ and $\gamma \in \mathscr{K}_{\infty}$ and a modulus $\Lambda$ with respect to $(\mathscr{E}, \mathscr{S})$ such that $\left|z\left(t, t_{0}, z_{0}, \delta\right)\right|_{\mathscr{E}_{0}} \leq \beta\left(\Lambda\left(z_{0}\right), t-t_{0}\right)+\gamma\left(\|\delta\|_{\left.t_{0}, t\right]}\right)$ for all initial states $z_{0}=z\left(t_{0}\right) \in \mathscr{S}$, all $\delta \in \mathscr{M} \mathscr{E} \mathscr{B}(\mathscr{D})$, all initial times $t_{0} \geq 0$, and all $t \geq t_{0}$, where $|p|_{\mathscr{E}_{0}}$ is the (standard Euclidean) distance from any point $p \in \mathscr{S}$ to $\mathscr{E}_{0}$ and $z\left(\cdot, t_{0}, z_{0}, \delta\right)$ is the trajectory for the system for the disturbance choice $\delta$ satisfying $z\left(t_{0}\right)=z_{0}$ which we require to remain in $\mathscr{S}$. Here $\mathscr{K} \mathscr{L}$ and $\mathscr{K}_{\infty}$ are the standard classes of comparison functions [8]. By a modulus with respect to $(\mathscr{E}, \mathscr{S})$, we mean a function $\Lambda: \mathscr{S} \rightarrow[0,+\infty)$ that is 0 at $\mathscr{E} 0$, positive at all other points in $\mathscr{S}$, and radially unbounded in the following sense: For each constant $K>0$, there is a constant $\delta_{K}>0$ such that $\Lambda(x) \geq K$ for all $x \in \mathscr{S}$ that satisfy either $\operatorname{dist}(x$, boundary $(\mathscr{S})) \leq \delta_{K}$ or $|x|_{\mathscr{E}_{0}} \geq 1 / \delta_{K}$. By an ISS Lyapunov function, we mean a proper positive definite function $V$ that admits a modulus $J$ with respect to $\left(\mathscr{E}_{0}, \mathscr{S}\right)$ and a function $\gamma \in \mathscr{K}_{\infty}$ such that $\dot{V} \leq-J(x)+\gamma(|\delta|)$ along all of the system trajectories for all $\delta \in \mathscr{M} \mathscr{E} \mathscr{B}(\mathscr{D})$.

Notice that ISS requires $\mathscr{S}$ to be robustly forwardly invariant for (20) and the disturbance set $\mathscr{D}$ [9], meaning all trajectories of (20) starting in $\mathscr{S}$ remain in $\mathscr{S}$ for all choices of $\delta \in \mathscr{M} \mathscr{E} \mathscr{B}(\mathscr{D})$. Therefore, $\mathscr{D}$ cannot be all of $\mathbb{R}^{2}$, since the trajectories of (20) must stay in $\mathscr{X}$. To see why restrictions on $\mathscr{D}$ are needed, take the initial state $\left(\rho_{1}(0), \zeta(0), \rho_{2}(0), \theta(0)\right)=\left(2 \rho_{c 1}, 0,2 \rho_{c 2}, 0\right) \in \mathscr{X}$ and the constant choices $\delta_{1}(t)=0$ and $\delta_{2}(t)=-\left(h_{1}^{\prime}\left(3 \rho_{c 1}\right)+\left\|\kappa_{n}\right\|_{\infty}+\right.$ $\mu)-\pi /\left(2 \min \left\{\rho_{c 1}, \rho_{c 2}\right\}\right)$. The corresponding trajectory of (20) satisfies $\left|\dot{\rho}_{i}(t)\right| \leq 1$ for all $t \geq 0$, so $\rho_{i}(t) \in\left[\rho_{c i}, 3 \rho_{c i}\right]$ and so also $h_{i}^{\prime}\left(\rho_{i}(t)\right) \geq 0$ for all $t \in\left[0, \min \left\{\rho_{c 1}, \rho_{c 2}\right\}\right]$ for $i=1,2$. Since $\kappa_{n}$ and $\kappa_{g}$ are nonpositive valued and $\alpha$ stays positive, this gives $\dot{\theta}(t) \leq h_{1}^{\prime}\left(3 \rho_{c 1}\right)+\left\|\kappa_{n}\right\|_{\infty}+\mu+\delta_{2}(t) \leq$ $-\pi /\left(2 \min \left\{\rho_{c 1}, \rho_{c 2}\right\}\right)$ for all $t \in\left[0, \min \left\{\rho_{c 1}, \rho_{c 2}\right\}\right]$ for which the trajectory is in $\mathscr{X}$. Hence, $\theta(t)$ reaches $-\pi / 2$ by time $t=\min \left\{\rho_{c 1}, \rho_{c 2}\right\}$, so the trajectory exits $\mathscr{X}$. This motivates:

Goal 1: Find bounded sets $\mathscr{S}_{i}$ containing the equilibrium $\mathscr{E}=\left(\rho_{c 1}, 0, \rho_{c 2}, 0\right)$ and satisfying $\cup_{i} \mathscr{S}_{i}=\mathscr{X}$, and sequences $\left\{\bar{\delta}_{1 i}\right\}$ and $\left\{\bar{\delta}_{2 i}\right\}$ of positive constants, such that these conditions hold for all $i$ : (G1) for all constants $a \in\left(0, \bar{\delta}_{1 i}\right)$ and $b \in\left(0, \bar{\delta}_{2 i}\right)$, the set $\mathscr{S}_{i}$ is robustly forwardly invariant for (20) and the disturbance set $\mathscr{D}=[-a, a] \times[-b, b]$ and (G2) if $a>\bar{\delta}_{1 i}$ or if $b>\bar{\delta}_{2 i}$, then the set $\mathscr{S}_{i}$ is not robustly forwardly invariant for (20) with the disturbance set $\mathscr{D}=$ $[-a, a] \times[-b, b]$.

Goal 1 is important because maintaining robust forward invariance ensures predictable tolerance and safety bounds that are robust to disturbances. Condition (G2) means that the disturbance bounds $\bar{\delta}_{1 i}$ and $\bar{\delta}_{2 i}$ are maximal for maintaining robust forward invariance of the $\mathscr{S}_{i}$ 's. We realized the 2D analog of Goal 1 in [9], where the $\mathscr{S}_{i}$ 's were 2D hexagons and there was only one scalar disturbance. This gave ISS on each $2 \mathrm{D}$ hexagon, but the 2D arguments do not carry over to $3 \mathrm{D}$. In this section, we obtain a 3D analog of the $2 \mathrm{D}$ robust forward invariance result that will be key to proving our ISS result. To this end, we assume in the rest of our work that:

Assumption 3: Assumption 2 holds, and $\lim _{\rho_{i} \rightarrow 0+} h_{i}^{\prime}\left(\rho_{i}\right)=$ $-\infty, \lim _{\rho_{i} \rightarrow+\infty} h_{i}^{\prime}\left(\rho_{i}\right)=+\infty$, and $h_{i}^{\prime \prime}\left(\rho_{c i}\right)>0$ hold for $i=1,2$. Also, the curvatures depend only on $Y=\left(\rho_{1}, \zeta, \rho_{2}, \theta\right)$.

The $h_{i}$ 's from (11) satisfy the requirements of Assumption 3 . We next define our robustly forwardly invariant sets. Fix any constants $\bar{\zeta} \in(0, \pi / 2)$ and $\bar{\theta} \in(0, \pi / 2)$. Take any quadruple $\left(\rho_{* 1}, \rho_{* 2}, K_{1}, K_{2}\right)$ of positive numbers. Let $H_{1}\left(\rho_{* 1}, \bar{\zeta}, K_{1}\right)$ be the closed region in the $\left(\rho_{1}, \zeta\right)$ plane that is enclosed by the hexagon having the vertices $A=$ $\left(\rho_{* 1}, 0\right), B=\left(\rho_{* 1}+\bar{\zeta} / \mu, \bar{\zeta}\right), C=\left(\rho_{* 1}+2 \bar{\zeta} / \mu+K_{1}, \bar{\zeta}\right)$, $D=\left(\rho_{* 1}+2 \bar{\zeta} / \mu+K_{1}, 0\right), E=\left(\rho_{* 1}+\bar{\zeta} / \mu+K_{1},-\bar{\zeta}\right)$, and $F=\left(\rho_{* 1},-\bar{\zeta}\right)$. Its legs $A B$ and $D E$ have slope $\mu$, and its other legs are horizontal or vertical. Let $H_{2}\left(\rho_{* 2}, \bar{\theta}, K_{2}\right)$ be the closed region in the $\left(\rho_{2}, \theta\right)$ plane that is enclosed by the hexagon with the vertices $A^{\prime}=\left(\rho_{* 2}, 0\right), B^{\prime}=\left(\rho_{* 2}, \bar{\theta}\right), C^{\prime}=$ $\left(\rho_{* 2}+\bar{\theta} /(\mu \cos (\bar{\zeta}))+K_{2}, \bar{\theta}\right), D^{\prime}=\left(\rho_{* 2}+2 \bar{\theta} /(\mu \cos (\bar{\zeta}))+\right.$ $\left.K_{2}, 0\right), \quad E^{\prime}=\left(\rho_{* 2}+2 \bar{\theta} /(\mu \cos (\bar{\zeta}))+K_{2},-\bar{\theta}\right)$, and $F^{\prime}=$ $\left(\rho_{* 2}+\bar{\theta} /(\mu \cos (\bar{\zeta})),-\bar{\theta}\right)$. Its legs $A^{\prime} F^{\prime}$ and $C^{\prime} D^{\prime}$ have slope $-\cos (\bar{\zeta}) \mu$, and its other legs are horizontal or vertical. See
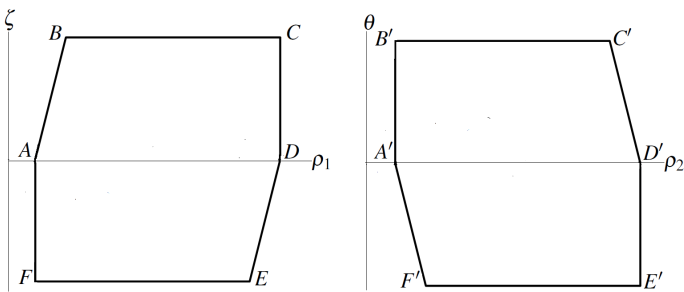

Fig. 1. Hexagononal Regions $H_{1}\left(\rho_{* 1}, \bar{\zeta}, K_{1}\right)$ and $H_{2}\left(\rho_{* 2}, \bar{\theta}, K_{2}\right)$ for Robustly Forwardly Invariant Set $H_{1}\left(\rho_{* 1}, \bar{\zeta}, K_{1}\right) \times H_{2}\left(\rho_{* 2}, \bar{\theta}, K_{2}\right)$

Fig. 1 where for simplicity we took $\bar{\zeta}=\bar{\theta}$ so both hexagons have the same height, and see Remark 2 for the motivation for choosing hexagonal regions instead of boxes. We define

$$
\begin{aligned}
\mathscr{Q}_{1}(Y)= & -\frac{\cos (\zeta) \kappa_{n}}{1-\kappa_{n} \rho_{1}-\kappa_{g} \rho_{2}} \frac{\sin ^{2}(\theta)}{\cos (\theta)}-\frac{\alpha \kappa_{g} \sin (\theta) \sin (\zeta)}{\cos (\theta)} \\
& +\frac{h_{1}^{\prime}\left(\rho_{1}\right) \cos (\zeta)}{\cos (\theta)}-\frac{\mu \sin (\zeta)}{\cos (\theta)} \text { and } \\
\mathscr{Q}_{2}(Y)= & -\frac{\kappa_{g} \cos ^{2}(\zeta) \cos (\theta)}{1-\kappa_{n} \rho_{1}-\kappa_{g} \rho_{2}}-\mu \cos (\zeta) \sin (\theta) \\
& +\left(-h_{1}^{\prime}\left(\rho_{1}\right)+\frac{\kappa_{n}}{1-\kappa_{n} \rho_{1}-\kappa_{g} \rho_{2}}\right) \sin (\zeta) \sin (\theta) \\
& +\left(-h_{2}^{\prime}\left(\rho_{2}\right)+\frac{\kappa_{g}}{1-\kappa_{n} \rho_{1}-\kappa_{g} \rho_{2}}\right) \cos (\theta)
\end{aligned}
$$

on our state space $\mathscr{X}$ from (8). Given any constants $\bar{\zeta} \in$ $(0, \pi / 2)$ and $\bar{\theta} \in(0, \pi / 2)$, we prove the following in [10]:

Lemma 1: Let $M_{i}>0$ be any constant for $i=1,2$. Then we can find constants $\bar{\rho}_{* 1} \in\left(0, \rho_{c 1}\right)$ and $\bar{K}_{1}>\rho_{c 1}$ such that for each pair $\left(\rho_{* 1}, K_{1}\right) \in\left(0, \bar{\rho}_{* 1}\right) \times\left(\bar{K}_{1},+\infty\right)$, there are constants $\mu>0, \rho_{* 2} \in\left(0, \rho_{c 2}\right)$, and $K_{2}>1$ such that: (C1) $\mathscr{Q}_{1}(Y)+\mu \sin (\zeta) \cos (\theta)>M_{1}$ (resp., $<-M_{1}$ ) for all $\left(\rho_{1}, \zeta\right) \in E D$ (resp., $\left.A B\right)$ and all $\left(\rho_{2}, \theta\right) \in H_{2}\left(\rho_{* 2}, \bar{\theta}, K_{2}\right)$, (C2) $\mathscr{Q}_{1}(Y)>M_{1}$ (resp., $<-M_{1}$ ) for all $\left(\rho_{1}, \zeta\right) \in F E$ 
(resp., $B C)$ and all $\left(\rho_{2}, \theta\right) \in H_{2}\left(\rho_{* 2}, \bar{\theta}, K_{2}\right),(\mathrm{C} 3) \mathscr{Q}_{2}(Y)+$ $\mu \cos (\bar{\zeta}) \sin (\theta)>M_{2}$ (resp., $<-M_{2}$ ) for all $\left(\rho_{2}, \theta\right) \in A^{\prime} F^{\prime}$ (resp., $\left.C^{\prime} D^{\prime}\right)$ and all $\left(\rho_{1}, \zeta\right) \in H_{1}\left(\rho_{* 1}, \bar{\zeta}, K_{1}\right)$ and (C4) $\mathscr{Q}_{2}(Y)>M_{2}$ (resp., $<-M_{2}$ ) for all $\left(\rho_{2}, \theta\right) \in F^{\prime} E^{\prime}$ (resp., $\left.B^{\prime} C^{\prime}\right)$ and all $\left(\rho_{1}, \zeta\right) \in H_{1}\left(\rho_{* 1}, \bar{\zeta}, K_{1}\right)$.

See [10] for details on the derivation of the $\bar{K}_{i}$ 's and $\bar{\rho}_{* i}$ 's and $\mu$. For any constants $M_{i}>0$, it follows that the constants

$$
\begin{aligned}
\Delta_{a}= & \min \left\{\left|\mathscr{Q}_{1}(Y)+\mu \sin (\zeta) \cos (\theta)\right|:\right. \\
& \left.\left(\rho_{1}, \zeta\right) \in A B \cup E D,\left(\rho_{2}, \theta\right) \in H_{2}\left(\rho_{* 2}, \bar{\theta}, K_{2}\right)\right\} \\
\Delta_{b}= & \min \left\{\left|\mathscr{Q}_{1}(Y)\right|:\left(\rho_{1}, \zeta\right) \in F E \cup B C,\right. \\
& \left.\left(\rho_{2}, \theta\right) \in H_{2}\left(\rho_{* 2}, \bar{\theta}, K_{2}\right)\right\} \\
\Delta_{c}= & \min \left\{\left|\mathscr{Q}_{2}(Y)+\mu \cos (\bar{\zeta}) \sin (\theta)\right|:\right. \\
& \left.\left(\rho_{2}, \theta\right) \in C^{\prime} D^{\prime} \cup A^{\prime} F^{\prime},\left(\rho_{1}, \zeta\right) \in H_{1}\left(\rho_{* 1}, \bar{\zeta}, K_{1}\right)\right\} \\
\Delta_{d}= & \min \left\{\left|\mathscr{Q}_{2}(Y)\right|:\left(\rho_{2}, \theta\right) \in B^{\prime} C^{\prime} \cup F^{\prime} E^{\prime},\right. \\
& \left.\left(\rho_{1}, \zeta\right) \in H_{1}\left(\rho_{* 1}, \bar{\zeta}, K_{1}\right)\right\}
\end{aligned}
$$

satisfy $\min \left\{\Delta_{a}, \Delta_{b}\right\} \geq M_{1}$ and $\min \left\{\Delta_{c}, \Delta_{d}\right\} \geq M_{2}$ when the requirements from Lemma 1 hold (since the minima are of continuous positive valued functions over compact sets).

We now have the tools to state our robust forward invariance result. For what follows, we say that a trajectory $Y(t)$ of (20) satisfying $Y(0) \in \mathscr{S}$ immediately exits $\mathscr{S}$ provided there is a constant $\varepsilon>0$ such that $Y(t) \notin \mathscr{S}$ for all $t \in(0, \varepsilon)$. Setting $\bar{\Delta}_{\zeta}=\min \left\{\Delta_{a}, \Delta_{b}\right\}$ and $\bar{\Delta}_{\theta}=\min \left\{\Delta_{c}, \Delta_{d}\right\}$, we have:

Theorem 2: Let $\left(M_{1}, M_{2}, \bar{\zeta}, \bar{\theta}, \rho_{* 1}, \rho_{* 2}, K_{1}, K_{2}, \mu\right)$ satisfy the requirements (C1)-(C4) of Lemma 1. Then: (a) For all constants $\delta_{* 1} \in\left(0, \bar{\Delta}_{\zeta}\right)$ and $\delta_{* 2} \in\left(0, \bar{\Delta}_{\theta}\right)$, the set $H_{1}\left(\rho_{* 1}, \bar{\zeta}, K_{1}\right) \times H_{2}\left(\rho_{* 2}, \bar{\theta}, K_{2}\right)$ is robustly forwardly invariant for (20) with disturbances valued in $\mathscr{D}=\left[-\delta_{* 1}, \delta_{* 1}\right] \times$ $\left[-\delta_{* 2}, \delta_{* 2}\right]$. (b) For each constant $\delta_{+}>\bar{\Delta}_{\zeta}\left(\right.$ resp., $\left.>\bar{\Delta}_{\theta}\right)$, there is a point $Y \in H_{1}\left(\rho_{* 1}, \bar{\zeta}, K_{1}\right) \times H_{2}\left(\rho_{* 2}, \bar{\theta}, K_{2}\right)$ such that the trajectory for (20) starting at $Y$ for one of the constant perturbations $\pm\left(\boldsymbol{\delta}_{+}, 0\right)$ (resp., $\left.\pm\left(0, \boldsymbol{\delta}_{+}\right)\right)$immediately exits $H_{1}\left(\rho_{* 1}, \bar{\zeta}, K_{1}\right) \times H_{2}\left(\rho_{* 2}, \bar{\theta}, K_{2}\right)$.

The proof of Theorem 2 uses Lemma 1 to show that the vector field for (20) points in along the entire boundary of $H_{1}\left(\rho_{* 1}, \bar{\zeta}, K_{1}\right) \times H_{2}\left(\rho_{* 2}, \bar{\theta}, K_{2}\right)$. For example, to show that the vector field points in along $A B$ (resp., $E D$ ), we show that $I_{1}\left(\rho_{1}, \zeta\right)=\zeta-\mu \rho_{1}$ satisfies $\dot{I}_{1}=\mathscr{Q}_{1}(Y)+\mu \sin (\zeta) \cos (\theta)+$ $\delta_{1}<0$ (resp., $>0$ ) along $A B$ (resp., $E D$ ) when $\left\|\delta_{1}\right\|_{\infty} \leq \bar{\Delta}_{\zeta}$. See [10] for details and the analysis of the other legs.

Remark 1: We can satisfy our conditions for a nested sequence of sets of the form $H_{1}\left(\rho_{* 1}, \bar{\zeta}, K_{1}\right) \times H_{2}\left(\rho_{* 2}, \bar{\theta}, K_{2}\right)$ that fill $\mathscr{X}$ and contain $\mathscr{E}=\left(\rho_{c 1}, 0, \rho_{c 2}, 0\right)$. This is done by choosing $\bar{\zeta}$ and $\bar{\theta}$ close enough to $\pi / 2$, reducing the $\rho_{* i}$ 's, and increasing the $K_{i}$ 's and $\mu$ so that the hexagons converge to larger and larger rectangles. Moreover, part (b) of Theorem 2 gives maximality of the disturbance bounds $\bar{\Delta}_{\zeta}$ and $\bar{\Delta}_{\theta}$. Therefore, we realized Goal 1. On the other hand, given any desired disturbance bound $\bar{M}$, we can choose the hexagon parameters to make $H_{1}\left(\rho_{* 1}, \bar{\zeta}, K_{1}\right) \times H_{2}\left(\rho_{* 2}, \bar{\theta}, K_{2}\right)$ robustly forwardly invariant under disturbances that are bounded by $\bar{M}$. This follows because we can make the constants $M_{1}$ and $M_{2}$ from Lemma 1 as big as we want.

Remark 2: We cannot replace our hexagons with boxes since for example the component pair $\left(\rho_{1}(t), \zeta(t)\right)$ from $(20)$ immediately exits the box $\left[\rho_{* 1}, \rho_{* 1}+2 \bar{\zeta} / \mu+K_{1}\right] \times[-\bar{\zeta}, \bar{\zeta}]$ through each point on the line segments $\left\{\rho_{* 1}\right\} \times(0, \bar{\zeta}]$ and $\left\{\rho_{* 1}+2 \bar{\zeta} / \mu+K_{1}\right\} \times[-\bar{\zeta}, 0)$ for all possible choices of the disturbance functions. A similar remark applies to $\left(\rho_{2}, \theta\right)$.

\section{Controller UnCERTAinty AND InPUt Delays}

We can view the disturbances $\delta_{i}$ as coming from additive uncertainties $\chi_{i}$ on the controls $a_{i}$ in (5) in the system [10]. We can also use Theorem 2 to get upper bounds on the input delays that can be introduced into the controls $a_{2}$ and $a_{3}$ without sacrificing the robust forward invariance, as follows. For simplicity, we assume that the curvatures are constant. We can write the perturbed closed loop system (20) in the form $\dot{Y}=\mathscr{F}(Y)+\left(0, \delta_{1}, 0, \delta_{2}\right)$ for a suitable function $\mathscr{F}$. Given any positive constant delays $\tau_{2}$ and $\tau_{3}$, we now replace the nonconstant controls $a_{i}(Y)$ from (5) by the time delayed controls $a_{i}\left(Y\left(t-\tau_{i}\right)\right)$ in the closed loop dynamics for $i=2$ and $i=3$. This gives the closed loop input delayed dynamics

$$
\dot{Y}=\mathscr{F}(Y)+\left(0, \Xi_{\zeta}\left(Y_{t}\right)+\tilde{\delta}_{1}, 0, \Xi_{\theta}\left(Y_{t}\right)+\tilde{\delta}_{2}\right)^{\top},
$$

where the functions $\tilde{\delta}_{1}$ and $\tilde{\delta}_{2}$ are additive uncertainties, the functions $Y_{t}$ are defined by $Y_{t}(s)=Y(s+t)$ for $-\max \left\{\tau_{2}, \tau_{3}\right\} \leq s \leq 0$, and the effects of the delays are captured by the terms

$$
\begin{aligned}
\Xi_{\zeta}\left(Y_{t}\right)= & -\frac{\cos (\zeta)}{\cos (\theta)}\left[a_{2}\left(\rho\left(t-\tau_{2}\right)\right)-a_{2}(\rho(t))\right] \\
\Xi_{\theta}\left(Y_{t}\right)= & \sin (\theta) \sin (\zeta)\left[a_{2}\left(\rho\left(t-\tau_{2}\right)\right)-a_{2}(\rho(t))\right] \\
& +\cos (\theta)\left[a_{3}\left(\rho\left(t-\tau_{3}\right)\right)-a_{3}(\rho(t))\right] .
\end{aligned}
$$

When $\mu>\max \{\bar{\zeta}, \bar{\theta} / \cos (\bar{\zeta})\}$, we have $\left|\Xi_{\zeta}\left(Y_{t}\right)\right| \leq$ $\mathscr{M}_{2} \tau_{2} / \cos (\bar{\theta})$ and $\left|\Xi_{\theta}\left(Y_{t}\right)\right| \leq \sin (\bar{\theta}) \sin (\bar{\zeta}) \mathscr{M}_{2} \tau_{2}+\mathscr{M}_{3} \tau_{3}$ on $H_{1}\left(\rho_{* 1}, \bar{\zeta}, K_{1}\right) \times H_{2}\left(\rho_{* 2}, \bar{\theta}, K_{2}\right)$, where $\mathscr{M}_{2}=\kappa_{n}^{2}+\left|\kappa_{n} \kappa_{g}\right|+$ ||$h_{1}^{\prime \prime}||_{\left[\rho_{* 1}, \rho_{* 1}+K_{1}+2\right]}$ and $\mathscr{M}_{3}=\kappa_{g}^{2}+\left|\kappa_{n} \kappa_{g}\right|+|| h_{2}^{\prime \prime}||_{\left[\rho_{* 2}, \rho_{* 2}+K_{2}+2\right]}$. Therefore, (23) is the special case of (20) with $\left(\delta_{1}, \delta_{2}\right)(t)=$ $\left(\Xi_{\zeta}\left(Y_{t}\right)+\tilde{\delta}_{1}(t), \Xi_{\theta}\left(Y_{t}\right)+\tilde{\delta}_{2}(t)\right)$, which satisfy

$$
\begin{aligned}
& \left\|\delta_{i}\right\|_{\infty} \leq\left\|\tilde{\delta}_{i}\right\|_{\infty}+ \\
& \max \left\{\tau_{2}, \tau_{3}\right\}\left(\left(\frac{1}{\cos (\bar{\theta})}+\sin (\bar{\zeta}) \sin (\bar{\theta})\right) \mathscr{M}_{2}+\mathscr{M}_{3}\right)
\end{aligned}
$$

for $i=1,2$. It follows from Theorem 2 that $H_{1}\left(\rho_{* 1}, \bar{\zeta}, K_{1}\right) \times$ $H_{2}\left(\rho_{* 2}, \bar{\theta}, K_{2}\right)$ is robustly forward invariant for (23) for all disturbances $\left(\tilde{\delta}_{1}, \tilde{\delta}_{2}\right)$ for which the right side of (25) is below $\delta_{* i}$ for $i=1$ and $i=2$. This shows [10]:

Theorem 3: Let $H_{1}\left(\rho_{* 1}, \bar{\zeta}, K_{1}\right) \times H_{2}\left(\rho_{* 2}, \bar{\theta}, K_{2}\right), \delta_{* 1}$, and $\delta_{* 2}$ satisfy the requirements from Theorem 2. Assume that the curvatures are negative constants and $\mu>$ $\max \{\bar{\zeta}, \bar{\theta} / \cos (\bar{\zeta})\}$. Choose any constants $\delta_{* * i}>0$ and any positive constants $\tau_{2}$ and $\tau_{3}$ such that

$$
\begin{aligned}
& \delta_{* * i}< \\
& \delta_{* i}-\max \left\{\tau_{2}, \tau_{3}\right\}\left(\left(\frac{1}{\cos (\bar{\theta})}+\sin (\bar{\zeta}) \sin (\bar{\theta})\right) \mathscr{M}_{2}+\mathscr{M}_{3}\right)
\end{aligned}
$$

for $i=1$ and $i=2$. Then $H_{1}\left(\rho_{* 1}, \bar{\zeta}, K_{1}\right) \times H_{2}\left(\rho_{* 2}, \bar{\theta}, K_{2}\right)$ is robustly forwardly invariant for (23) for all disturbances $\left(\tilde{\delta}_{1}, \tilde{\delta}_{2}\right)$ valued in $\mathscr{D}=\left[-\delta_{* * 1}, \delta_{* * 1}\right] \times\left[-\delta_{* * 2}, \delta_{* * 2}\right]$.

\section{ISS REVISITED}

We use the strict Lyapunov function to prove ISS of the perturbed dynamics (20) on each product $H_{1}\left(\rho_{* 1}, \bar{\zeta}, K_{1}\right) \times$ $H_{2}\left(\rho_{* 2}, \bar{\theta}, K_{2}\right)$ under the conditions of Theorem 2 with constant negative curvatures (but see [10] for adaptive cases 
where there are input delays, nonconstant curvatures, and identification of unknown control gains, which are based on converting our strict Lyapunov function $U$ from (14) into a barrier type strictified ISS Lyapunov-Krasovskii functional). In the next lemma, $|Y|_{\mathscr{E}}=|Y-\mathscr{E}|$ for all $Y \in \mathscr{O}$.

Lemma 2: If $U$ is a strict Lyapunov function for a system $\dot{Y}=F(Y)$ with equilibrium $\mathscr{E}$ on an open state space $\mathscr{O} \subseteq \mathbb{R}^{n}$, and $\mathscr{C}$ is a compact robustly forwardly invariant set for

$$
\dot{Y}=F(Y)+G(Y) \delta
$$

for some vector field $G$ with disturbances $\delta$ valued in some disturbance set $\mathscr{D}$ and $\mathscr{E} \in \mathscr{C}$, then $U$ is an ISS Lyapunov function for (26) on $\mathscr{C}$ with respect to disturbances valued in $\mathscr{D}$.

Proof: Let $\mathscr{M}=\max \left\{|Y|_{\mathscr{E}}: Y \in \mathscr{C}\right\}$ and choose the $\mathscr{K}_{\infty}$ functions $\underline{\alpha}(r)=[r /(1+\mathscr{M})] \min \{\min \{-\nabla U(Y) F(Y)$ : $\left.\min \{r, \mathscr{M}\} \leq|Y|_{\mathscr{E}} \leq \mathscr{M}\right\}, \min \left\{U(Y): \min \{r, \mathscr{M}\} \leq|Y|_{\mathscr{E}} \leq\right.$ $\mathscr{M}\}\}, \bar{\alpha}(r)=(1+r) \max \left\{U(Y):|Y|_{\mathscr{E}} \leq \min \{r, \mathscr{M}\}\right\}$, and $\alpha_{\infty}=\underline{\alpha} \circ \bar{\alpha}^{-1}$. Then $\underline{\alpha}\left(|Y|_{\mathscr{E}}\right) \leq-\nabla U(Y) F(Y), \underline{\alpha}\left(|Y|_{\mathscr{E}}\right) \leq$ $U(Y)$, and $\bar{\alpha}\left(|Y|_{\mathscr{E}}\right) \geq U(Y)$ hold for all $Y \in \mathscr{C}$. Set $\bar{c}=$ $\max \{|\nabla U(Y) G(Y)|: Y \in \mathscr{C}\}$. Then, $\dot{U} \leq-\alpha_{\infty}(U)+\bar{c}|\delta|$ holds along all trajectories of (26) in $\mathscr{C}$ with disturbances valued in $\mathscr{D}$, which is the ISS decay condition. Then standard ISS arguments provide functions $\beta_{0} \in \mathscr{K} \mathscr{L}$ and $\gamma_{0} \in \mathscr{K}_{\infty}$ such that $U(Y(t)) \leq \beta_{0}(U(Y(0)), t)+\gamma_{0}\left(|\delta|_{[0, t]}\right)$ along all trajectories of (26) in $\mathscr{C}$. Therefore, $\beta(r, s)=$ $\underline{\alpha}^{-1}\left(2 \beta_{0}(\bar{\alpha}(r), s)\right)$ and $\gamma(r)=\underline{\alpha}^{-1}\left(2 \gamma_{0}(r)\right)$ are such that $|Y(t)|_{\mathscr{E}} \leq \beta\left(|Y(0)|_{\mathscr{E}}, t\right)+\gamma\left(|\delta|_{[0, t]}\right)$ along all trajectories of (26) in $\mathscr{C}$ with disturbances valued in $\mathscr{D}$, so (26) is ISS on $\mathscr{C}$ for the disturbance set $\mathscr{D}$.

Specializing Lemma 2 to (20), $\mathscr{O}=\mathscr{X}$, and the sets $\mathscr{C}=$ $H_{1}\left(\rho_{* 1}, \bar{\zeta}, K_{1}\right) \times H_{2}\left(\rho_{* 2}, \bar{\theta}, K_{2}\right)$ from Theorem 2 gives:

Corollary 1: Let $H_{1}\left(\rho_{* 1}, \bar{\zeta}, K_{1}\right) \times H_{2}\left(\rho_{* 2}, \bar{\theta}, K_{2}\right), \delta_{* 1}$, and $\delta_{* 2}$ satisfy the requirements of Theorem 2 . Then (20) is ISS on $H_{1}\left(\rho_{* 1}, \bar{\zeta}, K_{1}\right) \times H_{2}\left(\rho_{* 2}, \bar{\theta}, K_{2}\right)$ for the disturbance set $\mathscr{D}=\left[-\boldsymbol{\delta}_{* 1}, \boldsymbol{\delta}_{* 1}\right] \times\left[-\boldsymbol{\delta}_{* 2}, \boldsymbol{\delta}_{* 2}\right]$.

To illustrate our ISS result, we simulated (20) using $\rho_{c 1}=$ $\rho_{c 2}=1$, the functions $h_{i}$ in (11) with $\bar{c}=0.05$, the constant curvatures $\kappa_{n}=\kappa_{g}=-0.1$, the parameters $\bar{\zeta}=\pi / 4, \bar{\theta}=$ $0.075 \pi, \mu=125, K_{1}=80, K_{2}=0.1, \rho_{* 1}=0.1$, and $\rho_{* 2}=$ 0.8 , and $\delta_{1}(t)=4 \sin (0.2 t)$ and $\delta_{2}(t)=0.1 \sin (t)$. We report our simulations in Fig. 2. Our simulations show convergence of the states to $(1,0,1,0)$ with an overshoot from the ISS estimate and so help validate our theory.

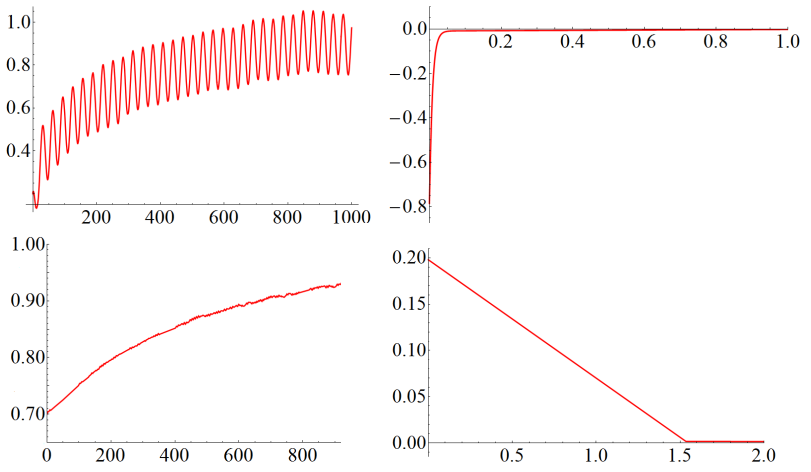

Fig. 2. Simulated Values of $\rho_{1}(t)$ (Upper Left), $\zeta(t)$ (Upper Right), $\rho_{2}(t)$ (Lower Left), and $\theta(t)$ (Lower Right) for Perturbed 3D Curve Tracking System (20) with $\delta(t)=(4 \sin (0.2 t), 0.1 \sin (t))$. Units: Meters and Seconds.

\section{CONCLUSIONS}

Curve tracking under state constraints, input delays and controller uncertainty is a challenging problem with important implications to the use of marine robots to detect pollution and other applications. Motivated by our deployment of marine robots in our search for residual pollutants from the Deepwater Horizon oil spill disaster, we found strict Lyapunov functions for a class of closed loop 3D curve tracking dynamics, and proved robust forward invariance of a class of hexagonal product sets. This made it possible to prove ISS under maximal disturbance bounds. We can also use our results to prove 3D adaptive tracking and parameter identification under unknown actuator gains, state constraints, actuator errors, and input delays [10].

\section{REFERENCES}

[1] A. Aguiar and J. Hespanha, "Trajectory-tracking and path-following of underactuated autonomous vehicles with parametric modeling uncertainty," IEEE Trans. Automat. Control, 52(8), pp. 1362-1379, 2007.

[2] D. Bresch-Pietri and M. Krstic, "Delay-adaptive predictor feedback for systems with unknown long actuator delay," IEEE Trans. Automat. Control, 55(9), pp. 2106-2112, 2010.

[3] K.D. Do, Z.P. Jiang, and J. Pan, "Robust adaptive path following of underactuated ships," Automatica, 40(6), pp. 929-944, 2004.

[4] E. Justh and P.S. Krishnaprasad, "Natural frames and interacting particles in three dimensions," in Proc. 44th IEEE Conf. on Decision and Control and European Control Conf., pp. 2841-2846, 2005.

[5] H. Khalil, Nonlinear Systems, Third Edition, Prentice Hall, Upper Saddle River, NJ, 2002.

[6] M. Krstic, Delay Compensation for Nonlinear, Adaptive, and PDE Systems, Birkhäuser Boston, Boston, MA, 2009.

[7] R. Lenain, B. Thuilot, C. Cariou, and P. Martinet, "High accuracy path tracking for vehicles in presence of sliding: Application to farm vehicle automatic guidance for agricultural tasks," Autonomous Robots, 21(1), pp. 79-97, 2006.

[8] M. Malisoff and F. Mazenc, Constructions of Strict Lyapunov Functions, Communications and Control Engineering Series, SpringerLondon Ltd., London, UK, 2009.

[9] M. Malisoff, F. Mazenc, and F. Zhang, "Stability and robustness analysis for curve tracking control using input-to-state stability," IEEE Trans. Automat. Control, 57(5), pp. 1320-1326, 2012.

[10] M. Malisoff and F. Zhang, "Robustness of adaptive control under time delays for three-dimensional curve tracking," preprint. https://www.math.lsu.edu/ malisoff/.

[11] A. Micaelli and C. Samson, "Trajectory tracking for unicycle-type and two-steering-wheels mobile robots," INRIA Report 2097, 1993.

[12] C. Samson, "Control of chained systems: Application to pathfollowing and time-varying point-stabilization of mobile robots," IEEE Trans. Automat. Control, 40(1), pp. 64-77, 1995.

[13] C. Woolsey and L. Techy, "Cross-track control of a slender, underactuated AUV using potential shaping," Ocean Engineering: Special Issue on AUVs, 26(1), pp. 82-91, 2009.

[14] X. Xiang, L. Lapierre, C. Liu, and B. Jouvencel, "Path tracking: combined path following and trajectory tracking for autonomous underwater vehicles," in Proc. IEEE/RSJ International Conf. on Intelligent Robots and Systems, pp. 3558-3563, 2011.

[15] F. Zhang, D.M. Fratantoni, D. Paley, J. Lund, and N. Leonard, "Control of coordinated patterns for ocean sampling," International J. Control, 80(7), pp. 1186-1199, 2007.

[16] F. Zhang, E. Justh, and P. Krishnaprasad, "Boundary following using gyroscopic control," in Proc. 43rd IEEE Conf. on Decision and Control, pp. 5204-5209, 2004.

[17] F. Zhang, E. Justh and P.S. Krishnaprasad, "Curve tracking and obstacle avoidance using gyroscopic control," in Recent Trends in Dynamical Systems: Proc. Conf. in Honor of Jürgen Scheurle, to appear.

[18] F. Zhang, A. O'Connor, D. Luebke, and P. Krishnaprasad, "Experimental study of curvature-based control laws for obstacle avoidance," in Proc. IEEE International Conf. on Robotics and Automation, pp. 3849-3854, 2004. 